\title{
Persistence in health behaviors among Medicare beneficiaries"
}

\author{
Bruce Stuart ${ }^{1,2}$, Amy Davidoff ${ }^{1,2}$, Francoise Pradel ${ }^{1,2}$, Ruth Lopert ${ }^{3}$, Thomas Shaffer $^{1,2}$, \\ Eberechukwu Onukwugha ${ }^{1,2}$, Franklin Hendrick ${ }^{1,2 \#}$, Jennifer Lloyd ${ }^{2}$ \\ ${ }^{1}$ The Peter Lamy Center on Drug Therapy and Aging, School of Pharmacy, University of Maryland, Baltimore, USA; \\ ${ }^{\#}$ Corresponding Author: fhend001@umaryland.edu \\ ${ }^{2}$ Graduate School, University of Maryland, Baltimore, USA \\ ${ }^{3}$ Department of Health Policy, School of Public Health \& Health Services, George Washington University, Washington DC, USA
}

Received 6 October 2011; revised 12 November 2011; accepted 13 December 2011

\section{ABSTRACT}

We examined persistence in seven common preventive health practices for a nationally representative sample of Medicare beneficiaries over 4-year observation periods. Six panels from the 19972005 Medicare Current Beneficiary Survey (MCBS) were used resulting in 13,913 unique individuals with ages ranging from below 65 (disabled) to over 80 years old. Persistence in behavior was defined as the proportion of the observation period beneficiaries participated in each activity. We estimated behavioral persistence as a function of baseline demographic, socioeconomic, and health characteristics using multivariate regression analysis. Beneficiaries were most persistent in smoking abstinence (81\% reported not smoking) and least persistent with routine exercise ( $47 \%$ reporting none). From multivariate regression results, there was greater persistence among beneficiaries who were married when compared to those living alone ( $p<0.01$ except for weekly exercise, $p<0.05$ and cholesterol screening, ns), with at least a high school education compared to no high school ( $p$ $<0.01$ for weekly exercise, prostate cancer screening, pap smear, $p<0.05$ for influenza vaccination and mammography, but ns for smoking cessation and cholesterol screening), and of higher income $(>300 \% \mathrm{FPL}$ compared to $<100 \% \mathrm{FPL}$ all $p<0.01$ ). Increasing age (greater than $80 \mathrm{com}$ pared to 65 - 69) was associated with increased compliance in influenza vaccination and smoking cessation $(p<0.01)$ while negatively associated with weekly exercise and cancer screenings $(p<0.01)$. Medicare beneficiaries are inconsistently persistent with common preventive health practices.

\footnotetext{
*Persistence in health behaviors among medicare beneficiaries.
}

Keywords: Behavior; Elderly; Medicare; Preventive Services

\section{INTRODUCTION}

Several studies have demonstrated the importance of healthy lifestyles, and primary and secondary preventive measures in reducing or delaying the burden of chronic health conditions, even among the elderly [1-6]. For example, several studies have examined the value of physical activity in preventing heart disease and colon cancer, limiting the effects of chronic diseases such as arthritis, and improving coordination and flexibility to help avoid falls and alleviate depression among older adults [1-4]. There is also evidence concerning smoking cessation, and for utilization of some primary prevention and disease screening services [5,6]. Historically, Medicare did not cover routine preventive services, but beginning with coverage of pneumococcal vaccination in 1981, the program has extended coverage to an increasing range of screening and vaccinations. Most recently, the Medicare Modernization Act of 2003 added a one-time "Welcome to Medicare" physical examination and health risk appraisal, and most vaccinations are covered under Medicare Part D plans. With passage of the Patient Protection and Affordable Care Act in 2010, all preventive services with a grade of A or B by the US Preventive Services Task Force (USPSTF) are available free to Medicare beneficiaries. While Medicare coverage of services is an important step, it is not the only factor to affect behaviors. Furthermore, the challenge for clinicians, program planners, and policy makers is how to encourage Medicare beneficiaries to initiate and maintain desired lifestyle and service use behaviors.

There is considerable published research on the characteristics of Medicare beneficiaries who use covered preventive services as well as other health practices. Influenza vaccination is perhaps the most thoroughly analyzed preventive health behavior among the elderly [7-12], but 
the research covers a broad spectrum of other health practices as well [13-20]. The general consensus from this body of research is that preventive services are underused by Medicare beneficiaries, particularly among disadvantaged segments of the population [11,16-18].

With rare exception, these studies provide point-in-time estimates and do not consider whether elderly individuals are persistent in their health behaviors over time $[12,19$, $21,22]$. Knowledge of persistence in health behaviors is important for two major reasons. First, the health returns from some behaviors-exercise and smoking avoidance for example-improve with persistence. Persistence in annual vaccinations also appears to increase the immune response to influenza vaccine $[23,24]$. A second reason is that some screening interventions such as mammography and cervical cancer screening are not recommended on an annual basis for this population (and in the very elderly may not be appropriate at all) $[25,26]$. Annual surveys thus tend to underestimate the true rate of adherence to guideline receipt of these tests.

This study had two aims: 1) to assess the persistence with which Medicare beneficiaries practice common health promotion and disease screening behaviors; and 2) to determine whether there are common factors that explain why some beneficiaries are more persistent in practicing healthy behaviors than others. We selected 7 common health behaviors for study, 2 measures of healthy lifestyle (routine exercise, smoking avoidance), 1 measure for disease prevention (influenza vaccination), and 4 measures of disease screening (cholesterol testing, mammography, cervical and prostate cancer screening). Persistence in each behavior was tracked over 4 years for a nationally representative sample of community-dwelling Medicare beneficiaries.

\section{METHODS}

\subsection{Data Source and Study Sample}

Data for the study were drawn from the Medicare Current Beneficiary Survey (MCBS) Access to Care (ATC) surveys from 1997 through 2005. The MCBS is a nationally representative rotating panel survey of the Medicare population conducted by the Centers for Medicare and Medicaid Services. Approximately 4500 beneficiaries are inducted into the MCBS survey each fall and are followed for up to three additional years. The sample is refreshed with a new induction cohort each year and an equal number are retired from the survey. The ATC survey captures a rich set of data on demographic and socioeconomic characteristics, health status (self-reported health, diseases, functioning), health behaviors, and access to care. Medicare claims with diagnostic codes are supplied with the MCBS files.

To assess behavioral persistence, we selected community-dwelling MCBS respondents who had complete annual
ATC surveys and survived their 4-year selection period. This process resulted in 6 cohorts (1997-2000, 1998-2001, etc.) representing 13,913 unique individuals. Because we required complete reporting for each behavior under study, persons who had missing values for behavioral questions of interest were excluded from the analyses of those behaviors. The MCBS initiated questions for two of our selected behaviors (exercise and cholesterol testing) in 2001, which limited the available samples for analyses of these behaviors. The study was approved by the University of Maryland Baltimore Institutional Review Board (IRB).

\subsection{Measures}

All 7 behaviors studied were measured based on selfreports during the fall ATC survey rounds. Question wording and rules for coding responses are provided in Table 1. Persistence was measured as the proportion of years a respondent indicated they practiced the behavior, with values ranging from 0 (no affirmative response in any year), 0.25 (behavior reported in 1 of the 4 years), through 0.50 , 0.75 , and 1.0 (behavior reported in all 4 years).

The exercise question in the MCBS is only asked on alternate years, which limited each respondent to 2 responses over the 4 years observation period. We coded persistence in this case as 0 (no activity in the 2 years in which the question was asked), 0.5 (1 year), and 1.0 (both years).

We included a wide array of explanatory variables in each behavioral persistence model to test for common factors expected to predict behavioral persistence. These variables included basic demographics (age, sex, race, living situation based on information about marital status and household composition, and acculturation as indicated by taking the MCBS in a language other than English), basis for program entitlement (under age 65 recipient of Social Security Disability Income (SSDI)), attitudes about seeking medical care, socioeconomic factors (educational attainment, income in relation to the Federal poverty level, presence of supplemental medical coverage), and heath status measures (self-reported health, body mass index, count of limitations in activities of daily living (ADL) [27], and count of comorbidities). The count of comorbidities is based on 189 hierarchical co-existing conditions (HCCs) derived from diagnostic codes in each beneficiary's annual Medicare claims. The HCC is used by the Centers for Medicare and Medicaid Services (CMS) to risk adjust capitation payments to Medicare Advantage plans, and is widely used as a comorbidity index in studies of the Medicare population [28-30]. Because several of the behaviors evaluated are associated with specific clinical indications either for or against their use, we included 17 self-reported disease and function indicators relevant to specific health behaviors. These behavior specific measures are listed in Table 1. 
Table 1. Measurement of persistence in healthy behaviors using MCBS panel data.

\begin{tabular}{|c|c|c|c|c|c|}
\hline Behavior & Question Wording & Response Measure & Frequency & Survey Years & Supplemental Control Variables \\
\hline $\begin{array}{l}\text { Routine } \\
\text { Exercise }\end{array}$ & $\begin{array}{l}\text { In a usual week, (do you/does } \mathrm{SP}^{\mathrm{a}} \text { ) } \\
\text { participate in any physical activities, } \\
\text { exercise, or sports such as bowling, } \\
\text { brisk walking, gardening, bicycling, } \\
\text { golf, swimming, or aerobics? }\end{array}$ & Yes/No & Biannual & $\begin{array}{l}2001,2003, \\
2005\end{array}$ & $\begin{array}{l}\text { Rheumatoid and osteoarthritis, } \\
\text { osteoporosis, and serious difficulty } \\
\text { or inability in walking or stooping }\end{array}$ \\
\hline $\begin{array}{l}\text { Smoking } \\
\text { Avoidance }\end{array}$ & $\begin{array}{l}\text { (Do you/Does SP) smoke cigarettes, } \\
\text { cigars, or pipe tobacco now? }\end{array}$ & Yes/No & Annual & $1997-2005$ & $\begin{array}{l}\text { Lung cancer, } \\
\text { emphysema/asthma/COPD }\end{array}$ \\
\hline $\begin{array}{l}\text { Influenza } \\
\text { Vaccination }\end{array}$ & $\begin{array}{l}\text { (Did you/Did SP) have a flu shot } \\
\text { for last winter? }\end{array}$ & Yes/No & Annual & $1997-2005$ & Emphysema/asthma/COPD \\
\hline $\begin{array}{l}\text { Cholesterol } \\
\text { Testing }\end{array}$ & $\begin{array}{l}\text { When was the most recent time } \\
\text { (you/SP) had (your/his/her) blood } \\
\text { cholesterol checked? }\end{array}$ & $\begin{array}{l}\text { If within } \\
\text { past } 12 \text { months }\end{array}$ & Annual & $2001-2005$ & $\begin{array}{l}\text { Diabetes, coronary heart disease, } \\
\text { stroke, myocardial infarction } \\
\text { "ever smoked" }\end{array}$ \\
\hline Mammography & $\begin{array}{l}\text { (Have you/Has SP) had a } \\
\text { mammogram or a breast X-ray } \\
\text { since [(PREV. SUPP. RD. INT. } \\
\text { DATE)/(TODAY'S DATE) a } \\
\text { year ago]? }\end{array}$ & Yes/No & Annual & $1997-2005$ & Breast cancer \\
\hline $\begin{array}{l}\text { Cervical } \\
\text { Cancer Screening }\end{array}$ & $\begin{array}{l}\text { (Have you/Has SP) had a Pap } \\
\text { smear test since [(PREV. SUPP. } \\
\text { RD. INT. DATE)/(TODAY'S } \\
\text { DATE) a year ago]? }\end{array}$ & Yes/No & Annual & $1997-2005$ & $\begin{array}{l}\text { Hysterectomy, uterine cancer, } \\
\text { and cancer of the cervix }\end{array}$ \\
\hline $\begin{array}{l}\text { Prostate } \\
\text { Cancer Screening }\end{array}$ & $\begin{array}{l}\text { - Have you/Has SP) had a digital } \\
\text { rectal examination (of the } \\
\text { prostate) since [(PREV. SUPP. } \\
\text { RD. INT. DATE)/(TODAY'S } \\
\text { DATE) a year ago]? } \\
\text { - Have you/Has SP) had a blood } \\
\text { test for detection of prostate } \\
\text { cancer, known as a PSA, since } \\
\text { [(PREV. SUPP. RD. INT. } \\
\text { DATE)/(TODAY'S DATE) } \\
\text { a year ago]? }\end{array}$ & $\begin{array}{l}\text { Yes to } \\
\text { either question }\end{array}$ & Annual & $1997-2005$ & Prostate cancer \\
\hline
\end{tabular}

${ }^{\mathrm{a}} \mathrm{SP}=$ survey participant.

We estimated behavior-specific models using 4-year proportional persistence measures as dependent variables and the explanatory variables measured at baseline (year 1). Each model was estimated using both a partial proportional odds model (PPOM) [31] and an ordinary least squares (OLS) linear probability model. The PPOM models used a generalized estimating equation algorithm to determine if the impact of a particular covariate was equivalent across the range of possible outcomes (e.g., whether the impact of being female was the same for individuals with 0 to 0.25 persistence in influenza vaccination compared to those with 0.75 to 1.0 persistence in this behavior). In all but a few instances, the PPOM models output consistent effects for each covariate across the spectrum of behavioral persistence, thus indicating linearity of response. The alternative OLS estimator presumes linearity of response and is preferred on efficiency grounds. We report only the OLS results here.

\section{RESULTS}

Table 2 reports the percentage of beneficiaries who reported each behavior from 0 to 4 years. For the 3 health promotion and disease prevention behaviors, persistence was highest in smoking avoidance $(81 \%$ reported not smoking at all, $11 \%$ always smoked, and $8 \%$ smoked at some point), followed by influenza vaccination ( $52 \%$ reported it each year), and lowest for exercise (only 24\% reported participating in weekly exercise in both years in which they were asked the question). There also was wide variation in persistence in disease screening and monitoring. Two-thirds of the sample reported cholesterol testing each year. Slightly more than $42 \%$ of women reported having $3(19 \%)$ or $4(23 \%)$ mammograms over the 4 -year period. Fewer women (26\%) reported having at least 3 Pap smears over the 4 years, but $42 \%$ reported having pap smears in 2 or more years. More than half of all men $(53 \%)$ reported a prostate cancer screen in 3 or 4 of the 
Table 2. Persistence in selected health behaviors for 4-year survivor cohorts of community-dwelling medicare beneficiaries, 19972005 (percentage and SE).

\begin{tabular}{|c|c|c|c|c|c|c|}
\hline \multirow{2}{*}{ Behavioral Variables } & \multirow{2}{*}{$\begin{array}{c}\text { Sample } \\
\text { Size }\end{array}$} & \multicolumn{5}{|c|}{ Percentage of Individuals Reporting Behavior } \\
\hline & & None & 1 year & 2 years & 3 years & 4 years \\
\hline \multicolumn{7}{|c|}{ Health Promotion and Disease Prevention } \\
\hline Routine weekly exercise $^{\mathrm{b}}$ & 6478 & $46.6(0.62)$ & $29.6(0.57)$ & $23.9(0.53)$ & NA & NA \\
\hline Smoking avoidance & 13,282 & $11.1(0.27)$ & $3.1(0.15)$ & $1.8(0.12)$ & $3.1(0.15)$ & $80.9(0.34)$ \\
\hline Influenza vaccination & 13,017 & $22.7(0.37)$ & $7.0(0.22)$ & $7.1(0.23)$ & $11.1(0.28)$ & $52.1(0.44)$ \\
\hline \multicolumn{7}{|c|}{ Disease Screening } \\
\hline Cholesterol testing ${ }^{\mathrm{c}}$ & 3756 & $4.5(0.34)$ & $4.2(0.33)$ & $8.5(0.46)$ & $15.2(0.59)$ & $67.6(0.76)$ \\
\hline Mammogram $^{d}$ & 7212 & $26.2(0.27)$ & $15.0(0.18)$ & $16.4(0.19)$ & $19.3(0.22)$ & $23.0(0.25)$ \\
\hline Pap smear $^{\mathrm{d}}$ & 7111 & $38.3(0.33)$ & $19.2(0.22)$ & $16.0(0.19)$ & $14.3(0.17)$ & $12.2(0.15)$ \\
\hline Prostate cancer screening ${ }^{\mathrm{e}}$ & 6102 & $18.1(0.24)$ & $13.9(0.20)$ & $15.3(0.21)$ & $22.7(0.29)$ & $30.0(0.34)$ \\
\hline
\end{tabular}

${ }^{\mathrm{b}}$ Panels 4,5 , and 6 only; ${ }^{\mathrm{c}}$ Panels 5 and 6 only; ${ }^{\mathrm{d}}$ Females only; ${ }^{\mathrm{e}}$ Males only.

years examined. The behaviors for which the largest proportions of beneficiaries reporting non participation were routine exercise (47\%) and cervical cancer screening (38\%).

Selected baseline characteristics of the full sample are reported in Table 3. These values reflect the average characteristics of community-dwelling Medicare beneficiaries with 4-year survival spans over the entire study period. Table 3 also presents mean persistence levels by beneficiary characteristic.

We find that persistence varies by beneficiary characteristic and by the nature of the behavior. For example, race is associated with the probability of behavioral persistence in exercise, with non-Hispanic whites having greater persistence (0.41) compared to non-Hispanic blacks (0.24), and Hispanics (0.27). Persistence in smoking avoidance also differs across race-ethnic groups, but the magnitudes are different $(0.86,0.78$, and 0.85 for whites, blacks and Hispanics respectively). Race does not affect persistence in cholesterol testing, and whereas persistence for blacks is lower for mammograms mammography and prostate cancer screening, blacks are actually the most persistent in receipt of pap smears with cervical cancer screening.

Table 4 presents results from the OLS multivariate regressions. The explained variance $\left(\mathrm{R}^{2}\right)$ for these models was reasonably high, varying between 0.17 for smoking avoidance to 0.36 for prostate cancer screening. Because of the way we coded the persistence variables, the model coefficients are read as marginal probabilities (e.g., beneficiaries $80+$ years old are 14 percentage points less persistent in routine exercise compared to beneficiaries aged 65 to 69$)$.

Persistence results were mixed for basic demographic factors. Increasing age was significantly associated with greater persistence in influenza vaccination and smoking avoidance, but lower persistence in exercise and cancer screening. Compared to non-Hispanic whites, non-Hispanic blacks were less persistent with routine exercise $(7 \%, \mathrm{p}<$ $0.01)$ and annual influenza vaccination $(9 \%, \mathrm{p}<0.01)$, but significantly more persistent in getting annual cholesterol tests $(5 \%, \mathrm{p}<0.01)$ and cancer screening $(7 \%$ for Pap smears and $4 \%$ for mammograms and prostate cancer screening, $\mathrm{p}<0.01$ in each case). Low acculturation was associated with greater persistence in smoking avoidance, but lesser persistence in influenza vaccination. Women were $6 \%$ less persistent in exercise and $8 \%$ more persistent in smoking avoidance compared with men $(\mathrm{p}<0.01)$. Compared with beneficiaries living alone, married persons were more persistent in all health behaviors except cholesterol testing, while those not married but living with others were either less persistent or similar to persons living alone. Poorer educational attainment was associated with lower persistence in exercise, influenza vaccination and cancer screening, while decreasing income was associated with lower rates of persistence in all behaviors. Supplemental medical insurance was associated with increased persistence in influenza vaccination, cholesterol testing, and mammography and cervical cancer screening - those services requiring supplemental payments.

Behavioral persistence also varied widely on baseline health, function, and BMI measures. Persistence in exercise, smoking avoidance, mammography, and cervical cancer screening declined significantly with worsening selfreported health, but rose with increasing comorbidity. The magnitude of these relationships was small $(7 \%$ or less) except for exercise, where beneficiaries in fair and poor health were $12 \%$ and $17 \%$, respectively, less persistent than individuals reporting very good to excellent 
Table 3. Mean persistence in health promotion and disease prevention behaviors and disease screening by individual characteristics $(\mathrm{n}=13,913)^{\mathrm{f}}$.

\begin{tabular}{|c|c|c|c|c|c|c|c|c|}
\hline Characteristics & $\begin{array}{l}\text { Percentage } \\
\text { Distribution }\end{array}$ & $\begin{array}{l}\text { Weekly } \\
\text { Exercise }\end{array}$ & $\begin{array}{l}\text { Smoking } \\
\text { Avoidance }\end{array}$ & $\begin{array}{l}\text { Influenza } \\
\text { Vaccination }\end{array}$ & $\begin{array}{l}\text { Cholesterol } \\
\text { Testing }\end{array}$ & Mammogram & Pap Smear & $\begin{array}{l}\text { Prostate Cancer } \\
\text { Screening }\end{array}$ \\
\hline Total sample & 100 & & & & & & & \\
\hline \multicolumn{9}{|l|}{ Age } \\
\hline$<65$ (SSDI disabled) & 18.5 & $0.26^{* *}$ & $0.63^{* *}$ & $0.43^{* *}$ & $0.73^{* *}$ & $0.41^{* *}$ & $0.47^{* *}$ & $0.30^{* *}$ \\
\hline $65-69$ & 22.3 & 0.50 & 0.84 & 0.65 & 0.87 & 0.60 & 0.43 & 0.66 \\
\hline $70-74$ & 17.1 & 0.45 & 0.89 & 0.71 & 0.86 & 0.56 & 0.38 & 0.70 \\
\hline $75-79$ & 17.9 & 0.43 & 0.93 & 0.75 & 0.86 & 0.53 & 0.33 & 0.69 \\
\hline $80+$ & 24.2 & 0.31 & 0.95 & 0.74 & 0.87 & 0.37 & 0.23 & 0.63 \\
\hline \multicolumn{9}{|l|}{ Sex } \\
\hline Female & 56.1 & $0.35^{* *}$ & $0.89^{* *}$ & $0.66^{*}$ & 0.84 & 0.50 & 0.36 & N/A \\
\hline Male & 43.9 & 0.44 & 0.81 & 0.65 & 0.84 & N/A & N/A & 0.58 \\
\hline \multicolumn{9}{|l|}{ Race/ethnicity } \\
\hline White/non-Hispanic & 80.6 & $0.41^{* *}$ & $0.86^{* *}$ & $0.69^{* *}$ & 0.84 & $0.51^{* *}$ & $0.35^{* *}$ & $0.61^{* *}$ \\
\hline Black/non-Hispanic & 9.9 & 0.24 & 0.78 & 0.49 & 0.84 & 0.45 & 0.40 & 0.47 \\
\hline Hispanic & 6.2 & 0.27 & 0.85 & 0.49 & 0.84 & 0.41 & 0.35 & 0.46 \\
\hline Other & 3.4 & 0.38 & 0.82 & 0.63 & 0.85 & 0.39 & 0.33 & 0.54 \\
\hline \multicolumn{9}{|l|}{ Living situation } \\
\hline Married with spouse & 49.7 & $0.45^{* *}$ & $0.88^{* *}$ & $0.71^{* *}$ & $0.86^{* *}$ & $0.58^{* *}$ & $0.42^{* *}$ & $0.66^{* *}$ \\
\hline $\begin{array}{l}\text { Not married and lives with } \\
\text { family }\end{array}$ & 8.7 & 0.26 & 0.82 & 0.56 & 0.85 & 0.50 & 0.32 & 0.50 \\
\hline $\begin{array}{l}\text { Note married and lives with } \\
\text { others }\end{array}$ & 9.9 & 0.30 & 0.73 & 0.48 & 0.74 & 0.35 & 0.34 & 0.28 \\
\hline Lives alone & 37.7 & 0.35 & 0.84 & 0.65 & 0.83 & 0.47 & 0.31 & 0.51 \\
\hline \multicolumn{9}{|l|}{ Culture integration } \\
\hline MCBS survey not in English & 3.4 & $0.21^{* *}$ & $0.89^{* *}$ & $0.44^{* *}$ & 0.89 & $0.39^{* *}$ & 0.34 & $0.50^{* *}$ \\
\hline MCBS survey in English & 96.6 & 0.39 & 0.85 & 0.66 & 0.84 & 0.50 & 0.36 & 0.58 \\
\hline \multicolumn{9}{|l|}{ Educational attainment } \\
\hline No high school education & 17.6 & $0.25^{* *}$ & $0.85^{* *}$ & $0.59^{* *}$ & $0.84^{* *}$ & $0.39^{* *}$ & $0.27^{* *}$ & $0.48^{* *}$ \\
\hline Some high school & 17.0 & 0.29 & 0.80 & 0.60 & 0.81 & 0.43 & 0.31 & 0.51 \\
\hline High school graduate & 31.2 & 0.37 & 0.85 & 0.66 & 0.83 & 0.51 & 0.37 & 0.56 \\
\hline >High school education & 33.5 & 0.50 & 0.88 & 0.72 & 0.87 & 0.57 & 0.42 & 0.69 \\
\hline \multicolumn{9}{|l|}{$\begin{array}{l}\text { Income in relation to poverty } \\
\text { line }\end{array}$} \\
\hline$<100 \% \mathrm{FPL}$ & 19.2 & $0.25^{* *}$ & $0.76^{* *}$ & $0.51^{* *}$ & $0.79^{* *}$ & $0.37^{* *}$ & $0.31^{* *}$ & $0.33^{* *}$ \\
\hline $101 \%-150 \%$ FPL & 32.1 & 0.30 & 0.83 & 0.63 & 0.83 & 0.44 & 0.32 & 0.50 \\
\hline $150 \%-300 \%$ FPL & 19.2 & 0.42 & 0.87 & 0.70 & 0.86 & 0.54 & 0.36 & 0.64 \\
\hline $300 \%$ FPL & 29.3 & 0.55 & 0.91 & 0.75 & 0.88 & 0.63 & 0.44 & 0.73 \\
\hline \multicolumn{9}{|l|}{ Aged $65+$ former SSDI } \\
\hline Yes & 24.3 & $0.25^{* *}$ & $0.68^{* *}$ & $0.48^{* *}$ & $0.78^{* *}$ & $0.43^{* *}$ & $0.43^{* *}$ & $0.37^{* *}$ \\
\hline No & 75.7 & 0.43 & 0.90 & 0.71 & 0.86 & 0.51 & 0.34 & 0.67 \\
\hline $\begin{array}{l}\text { Any supplemental medical } \\
\text { insurance }\end{array}$ & 90.5 & $0.39^{* *}$ & $0.86^{* *}$ & $0.68^{* *}$ & $0.85^{* *}$ & $0.51^{* *}$ & $0.37^{* *}$ & $0.60^{* *}$ \\
\hline Always try to avoid doctor visit & 31.6 & $0.33^{* *}$ & $0.80^{* *}$ & $0.56^{* *}$ & $0.78^{* *}$ & $0.39^{* *}$ & $0.30^{* *}$ & $0.49^{* *}$ \\
\hline Self-reported health status & & & & & & & & \\
\hline
\end{tabular}




\section{Continued}

\begin{tabular}{|c|c|c|c|c|c|c|c|c|}
\hline Very good to excellent & 41.2 & $0.52^{* *}$ & $0.90^{* *}$ & $0.68^{* *}$ & 0.83 & $0.52^{* *}$ & 0.37 & $0.64^{* *}$ \\
\hline Good & 29.5 & 0.37 & 0.86 & 0.68 & 0.85 & 0.50 & 0.35 & 0.60 \\
\hline Fair & 19.6 & 0.25 & 0.79 & 0.63 & 0.86 & 0.45 & 0.34 & 0.53 \\
\hline Poor & 9.6 & 0.13 & 0.71 & 0.56 & 0.84 & 0.43 & 0.36 & 0.44 \\
\hline \multicolumn{9}{|l|}{ Body Mass Index (BMI) } \\
\hline Underweight $(<18.5)$ & 2.5 & $0.30^{* *}$ & $0.70^{* *}$ & $0.58^{* *}$ & $0.78^{* *}$ & $0.31^{* *}$ & $0.26^{* *}$ & $0.41^{* *}$ \\
\hline Normal (18.5 - 24.9) & 36.0 & 0.43 & 0.83 & 0.66 & 0.82 & 0.49 & 0.35 & 0.55 \\
\hline Overweight (25.0 - 29.9) & 36.4 & 0.41 & 0.87 & 0.68 & 0.86 & 0.53 & 0.37 & 0.62 \\
\hline Obese Class I (30.0 - 34.9) & 15.2 & 0.34 & 0.87 & 0.66 & 0.86 & 0.51 & 0.37 & 0.59 \\
\hline Obese Class II (35.0 - 39.9) & 4.5 & 0.25 & 0.85 & 0.61 & 0.86 & 0.50 & 0.36 & 0.54 \\
\hline Obese Class III $(\geq 40)$ & 2.7 & 0.20 & 0.86 & 0.54 & 0.82 & 0.44 & 0.39 & 0.39 \\
\hline \multicolumn{9}{|l|}{ Census region (\%) } \\
\hline South & 40.5 & $0.35^{* *}$ & $0.84^{* *}$ & $0.64^{* *}$ & $0.86^{* *}$ & 0.49 & $0.37^{* *}$ & 0.58 \\
\hline East & 19.2 & 0.35 & 0.86 & 0.64 & 0.86 & 0.49 & 0.36 & 0.60 \\
\hline Midwest & 25.4 & 0.42 & 0.86 & 0.68 & 0.82 & 0.50 & 0.33 & 0.58 \\
\hline West & 14.8 & 0.46 & 0.85 & 0.69 & 0.82 & 0.50 & 0.36 & 0.58 \\
\hline \multicolumn{9}{|l|}{$\begin{array}{l}\text { Panel year (year of induction } \\
\text { into the MCBS survey) }\end{array}$} \\
\hline 1997 & 17.5 & NA & 0.84 & 0.64 & NA & $0.45^{* *}$ & 0.35 & $0.41^{* *}$ \\
\hline 1998 & 16.9 & NA & 0.84 & 0.66 & NA & 0.48 & 0.35 & 0.58 \\
\hline 1999 & 16.6 & NA & 0.85 & 0.65 & NA & 0.51 & 0.37 & 0.61 \\
\hline 2000 & 16.5 & $0.39^{* *}$ & 0.85 & 0.67 & NA & 0.53 & 0.36 & 0.63 \\
\hline
\end{tabular}

${ }_{\mathrm{f}}$ Mean was calculated by different sample size in each individual persistency measurement. ANOVA test for each individual characteristics and persistency measurement, ${ }^{*} \mathrm{p}<0.05,{ }^{* *} \mathrm{p}<0.01$.

Table 4. Regression results from OLS models predicting persistence of selected health behaviors for 4-year survivor cohorts of community-dwelling medicare beneficiaries, 1997-2005 ( $\mathrm{n}=13,913$, standard errors in parentheses).

\begin{tabular}{|c|c|c|c|c|c|c|c|}
\hline \multirow[b]{2}{*}{ Independent Variables } & \multicolumn{3}{|c|}{ Health Promotion and Disease Prevention } & \multicolumn{4}{|c|}{ Disease Screening } \\
\hline & $\begin{array}{l}\text { Weekly } \\
\text { Exercise }\end{array}$ & $\begin{array}{l}\text { Smoking } \\
\text { Avoidance }\end{array}$ & $\begin{array}{l}\text { Influenza } \\
\text { Vaccination }\end{array}$ & $\begin{array}{l}\text { Cholesterol } \\
\text { Testing }\end{array}$ & Mammogram & Pap Smear & $\begin{array}{c}\text { Prostate Cancer } \\
\text { Screening }\end{array}$ \\
\hline Intercept & $0.71(0.03)^{* *}$ & $0.73(0.02)^{* *}$ & $0.46(0.02)^{* *}$ & $0.70(0.03)^{* *}$ & $0.41(0.02)^{* *}$ & $0.38(0.02)^{* *}$ & $0.43(0.02)^{* *}$ \\
\hline \multicolumn{8}{|l|}{ Age } \\
\hline <65 (SSDI disabled) & $0.01(0.02)$ & $-0.16(0.01)^{* *}$ & $-0.16(0.02)^{* *}$ & $-0.12(0.02)^{* *}$ & $-0.07(0.02)^{* *}$ & $0.11(0.02)^{* *}$ & $-0.21(0.02)^{* *}$ \\
\hline $65-69$ & Ref & Ref & Ref & Ref & Ref & Ref & Ref \\
\hline $70-74$ & $-0.04(0.01)^{* *}$ & $0.05(0.01)^{* *}$ & $0.04(0.01)^{* *}$ & $-0.02(0.01)$ & $-0.05(0.01)^{* *}$ & $-0.05(0.01)^{* *}$ & $0.02(0.01)$ \\
\hline $75-79$ & $-0.06(0.01)^{* *}$ & $0.09(0.01)^{* *}$ & $0.07(0.01)^{* *}$ & $-0.02(0.01)$ & $-0.09(0.01)^{* *}$ & $-0.10(0.01)^{* *}$ & $-0.01(0.01)$ \\
\hline $80+$ & $-0.14(0.01)^{* *}$ & $0.13(0.01)^{* *}$ & $0.07(0.01)^{* *}$ & $-0.01(0.01)$ & $-0.21(0.01)^{* *}$ & $-0.18(0.01)^{* *}$ & $-0.06(0.01)^{* *}$ \\
\hline \multicolumn{8}{|l|}{ Sex } \\
\hline Female & $-0.06(0.01)^{* *}$ & $0.08(0.01)^{* *}$ & $0.00(0.01)$ & $-0.01(0.01)$ & - & - & - \\
\hline Male & Ref & Ref & Ref & Ref & - & - & - \\
\hline \multicolumn{8}{|l|}{ Race/ethnicity } \\
\hline White/non-Hispanic & Ref & Ref & Ref & Ref & Ref & Ref & Ref \\
\hline Black/non-Hispanic & $-0.07(0.02)^{* *}$ & $0.00(0.01)$ & $-0.09(0.01)^{* *}$ & $0.05(0.02)^{* *}$ & $0.04(0.01)^{*}$ & $0.07(0.01)^{* *}$ & $0.04(0.01)^{* *}$ \\
\hline Hispanic & $-0.02(0.02)$ & $0.03(0.01)$ & $-0.05(0.02)^{* *}$ & $0.01(0.02)$ & $-0.02(0.02)$ & $0.00(0.02)$ & $-0.02(0.02)$ \\
\hline
\end{tabular}




\section{Continued}

\begin{tabular}{|c|c|c|c|c|c|c|c|}
\hline Other & $0.01(0.02)$ & $0.01(0.02)$ & $-0.01(0.02)$ & $0.04(0.02)$ & $-0.05(0.02)^{*}$ & $0.01(0.02)$ & $0.00(0.02)$ \\
\hline \multicolumn{8}{|l|}{ Living Situation } \\
\hline Married with spouse & $0.02(0.01)^{*}$ & $0.06(0.01)^{* *}$ & $0.04(0.01)^{* *}$ & $0.02(0.01)$ & $0.04(0.01)^{* *}$ & $0.05(0.01)^{* *}$ & $0.06(0.01)^{* *}$ \\
\hline Not married and lives with family & $0.01(0.02)$ & $-0.0(0.01)$ & $-0.04(0.01)^{* *}$ & $0.02(0.02)$ & $-0.04(0.01)^{* *}$ & $-0.01(0.01)$ & $0.01(0.02)$ \\
\hline Not married and lives with others & $0.01(0.02)$ & $0.04(0.01)^{* *}$ & $-0.01(0.01)$ & $-0.02(0.02)$ & $-0.07(0.02)^{* *}$ & $-0.03(0.02)^{*}$ & $-0.07(0.02)^{* *}$ \\
\hline Lives alone & Ref & Ref & Ref & Ref & Ref & Ref & Ref \\
\hline \multicolumn{8}{|l|}{ Culture integration } \\
\hline MCBS survey not in English & $-0.05(0.03)$ & $0.06(0.02)^{* *}$ & $-0.14(0.02)^{* *}$ & $0.05(0.03)$ & $-0.01(0.03)$ & $0.02(0.03)$ & $0.03(0.03)$ \\
\hline MCBS survey in English & Ref & Ref & Ref & Ref & Ref & Ref & Ref \\
\hline \multicolumn{8}{|l|}{ Educational attainment } \\
\hline No high school education & $-0.07(0.02)^{* *}$ & $0.01(0.01)$ & $-0.03(0.01)^{*}$ & $-0.02(0.02)$ & $-0.04(0.01)^{*}$ & $-0.06(0.01)^{* *}$ & $-0.07(0.01)^{* *}$ \\
\hline Some high school & $-0.09(0.01)^{* *}$ & $-0.02(0.01)^{* *}$ & $-0.03(0.01)^{* *}$ & $-0.02(0.01)$ & $-0.04(0.01)^{* *}$ & $-0.04(0.01)^{* *}$ & $-0.04(0.01)^{* *}$ \\
\hline High school graduate & $-0.07(0.01)^{* *}$ & $-0.00(0.01)$ & $-0.01(0.01)$ & $-0.01(0.01)$ & $-0.02(0.01)$ & $-0.02(0.01)^{*}$ & $-0.03(0.01)^{* *}$ \\
\hline High school education & Ref & Ref & Ref & Ref & Ref & Ref & Ref \\
\hline \multicolumn{8}{|l|}{ Income in relation to poverty line } \\
\hline$<100 \%$ FPL & $-0.10(0.02)^{* *}$ & $-0.06(0.01)^{* *}$ & $-0.07(0.01)^{* *}$ & $-0.05(0.02)^{* *}$ & $-0.12(0.01)^{* *}$ & $-0.08(0.01)^{* *}$ & $-0.17(0.01)^{* *}$ \\
\hline $101 \%-150 \%$ FPL & $-0.10(0.01)^{* *}$ & $-0.04(0.01)^{* *}$ & $-0.04(0.01)^{* *}$ & $-0.03(0.01)^{*}$ & $-0.09(0.01)^{* *}$ & $-0.05(0.01)^{* *}$ & $-0.10(0.01)^{* *}$ \\
\hline $150 \%-300 \%$ FPL & $-0.06(0.01)^{* *}$ & $-0.03(0.01)^{* *}$ & $-0.02(0.01)^{* *}$ & $-0.02(0.01)$ & $-0.05(0.01)^{* *}$ & $-0.04(0.01)^{* *}$ & $-0.04(0.01)^{* *}$ \\
\hline $300 \%$ FPL & Ref & Ref & Ref & Ref & Ref & Ref & Ref \\
\hline \multicolumn{8}{|l|}{ Aged $65+$ former SSDI } \\
\hline Yes & $-0.07(0.02)^{* *}$ & $-0.00(0.01)$ & $0.01(0.02)$ & $0.01(0.02)$ & $0.01(0.02)$ & $0.01(0.02)$ & $-0.00(0.02)$ \\
\hline No & Ref & Ref & Ref & Ref & Ref & Ref & Ref \\
\hline $\begin{array}{l}\text { Any supplemental medical } \\
\text { insurance }\end{array}$ & $0.03(0.02)$ & $0.02(0.01)$ & $0.08(0.01)^{* *}$ & $0.05(0.02)^{* *}$ & $0.12(0.02)^{* *}$ & $0.07(0.02)^{* *}$ & $0.02(0.01)$ \\
\hline Always try to avoid doctor visit & $-0.03(0.01)^{* *}$ & $-0.05(0.01)^{* *}$ & $-0.09(0.01)^{* *}$ & $-0.06(0.01)^{* *}$ & $-0.10(0.01)^{* *}$ & $-0.06(0.01)^{* *}$ & $-0.06(0.01)^{* *}$ \\
\hline \multicolumn{8}{|l|}{ Self-reported health status } \\
\hline Very good to excellent & Ref & Ref & Ref & Ref & Ref & Ref & Ref \\
\hline Good & $-0.08(0.01)^{* *}$ & $-0.03(0.01)^{* *}$ & $0.01(0.01)$ & $0.02(0.01)$ & $-0.03(0.01)^{* *}$ & $-0.03(0.01)^{* *}$ & $-0.01(0.01)$ \\
\hline Fair & $-0.12(0.01)^{* *}$ & $-0.05(0.01)^{* *}$ & $0.01(0.01)$ & $0.01(0.01)$ & $-0.05(0.01)^{* *}$ & $-0.05(0.01)^{* *}$ & $0.00(0.01)$ \\
\hline Poor & $-0.17(0.02)^{* *}$ & $-0.08(0.01)^{* *}$ & $0.01(0.01)$ & $0.04(0.02)^{*}$ & $-0.07(0.02)^{* *}$ & $-0.07(0.02)^{* *}$ & $0.00(0.02)$ \\
\hline \multicolumn{8}{|l|}{ Body mass index (BMI) } \\
\hline Underweight $(<18.5)$ & $-0.05(0.03)$ & $-0.12(0.02)^{* *}$ & $-0.03(0.02)$ & $-0.01(0.03)$ & $-0.11(0.02)^{* *}$ & $-0.05(0.02)^{*}$ & $0.00(0.03)$ \\
\hline Normal (18.5 - 24.9) & Ref & Ref & Ref & Ref & Ref & Ref & Ref \\
\hline Overweight (25.0 - 29.9) & $-0.03(0.01)^{*}$ & $0.06(0.01)^{* *}$ & $0.02(0.01)^{* *}$ & $0.03(0.01)^{* *}$ & $0.04(0.01)^{* *}$ & $0.01(0.01)$ & $0.03(0.01)^{* *}$ \\
\hline Obese Class I (30.0 - 34.9) & $-0.04(0.01)^{* *}$ & $0.08(0.01)^{* *}$ & $0.03(0.01)^{* *}$ & $0.04(0.01)^{* *}$ & $0.03(0.01)^{*}$ & $0.01(0.01)$ & $0.05(0.01)^{* *}$ \\
\hline Obese Class II (35.0 - 39.9) & $-0.08(0.02)^{* *}$ & $0.10(0.01)^{* *}$ & $0.01(0.02)$ & $0.02(0.02)$ & $0.01(0.02)$ & $-0.02(0.02)$ & $0.04(0.02)$ \\
\hline Obese Class III $(\geq 40)$ & $-0.05(0.03)$ & $0.18(0.02)^{* *}$ & $0.01(0.02)$ & $0.03(0.02)$ & $0.01(0.02)$ & $-0.01(0.02)$ & $-0.01(0.03)$ \\
\hline \multicolumn{8}{|l|}{ Census region } \\
\hline South & $0.02(0.01)$ & $0.01(0.01)$ & $0.03(0.01)^{* *}$ & $0.00(0.01)$ & $0.01(0.01)$ & $0.02(0.01)$ & $-0.01(0.01)$ \\
\hline East & Ref & Ref & Ref & Ref & Ref & Ref & Ref \\
\hline Midwest & $0.05(0.01)^{* *}$ & $0.01(0.01)$ & $0.02(0.01)^{*}$ & $-0.03(0.01)^{*}$ & $0.01(0.01)$ & $-0.02(0.01)^{*}$ & $-0.03(0.01)^{* *}$ \\
\hline West & $0.07(0.02)^{* *}$ & $0.01(0.01)$ & $0.04(0.01)^{* *}$ & $-0.03(0.02)$ & $-0.01(0.01)$ & $-0.02(0.01)$ & $-0.03(0.01)^{*}$ \\
\hline \multicolumn{8}{|l|}{$\begin{array}{l}\text { Panel year (year of induction into } \\
\text { the MCBS survey) }\end{array}$} \\
\hline 1997 & NA & Ref & Ref & NA & Ref & Ref & Ref \\
\hline 1998 & NA & $-0.01(0.01)$ & $0.00(0.01)$ & NA & $0.02(0.01)$ & $-0.00(0.01)$ & $0.17(0.01)^{* *}$ \\
\hline 1999 & NA & $-0.00(0.01)$ & $-0.01(0.01)$ & NA & $0.05(0.01)^{* *}$ & $0.01(0.01)$ & $0.18(0.01)^{* *}$ \\
\hline 2000 & Ref & $-0.00(0.01)$ & $0.00(0.01)$ & NA & $0.06(0.01)^{* *}$ & $0.02(0.01)$ & $0.20(0.01)^{* *}$ \\
\hline 2001 & $0.05(0.01)^{* *}$ & $-0.00(0.01)$ & $-0.01(0.01)$ & Ref & $0.02(0.01)$ & $-0.01(0.01)$ & $0.19(0.01)^{* *}$ \\
\hline 2002 & $-0.06(0.01)^{* *}$ & $0.01(0.01)$ & $-0.01(0.01)$ & $0.03(0.01)^{* *}$ & $0.02(0.01)$ & $-0.00(0.01)$ & $0.21(0.01)^{* *}$ \\
\hline $\mathrm{R}^{2}$ & 0.25 & 0.17 & 0.18 & 0.19 & 0.23 & 0.19 & 0.36 \\
\hline
\end{tabular}

${ }^{*} \mathrm{p}<0.05 ;{ }^{* *} \mathrm{p}<0.01$; Source: Medicare Current Beneficiary Surveys, 1997-2005. 
health status $\mathrm{p}<0.01$ ). Beneficiaries who were overweight or with class I obesity were significantly more persistent with every health behavior except exercise and cervical cancer screening, compared with those with normal weight. Higher BMIs (class II and III obesity) were associated with more persistent smoking avoidance but less persistent exercise.

Of all the associations, two factors - having low income and avoiding physician visits - were the most consistent predictors of non-persistence across all 7 behaviors. Beneficiaries at or below the Federal poverty line at baseline were $11 \%$ less persistent with routine exercise compared to those at $300 \%$ or more above the FPL. These poorest beneficiaries were also $8 \%$ less persistent in getting annual flu shots and between $8 \%$ and $17 \%$ less likely to get annual cancer screening (all significant at $\mathrm{p}>0.01$ ).

\section{DISCUSSION}

These findings paint a decidedly mixed picture regarding the health habits of Medicare beneficiaries. Overall, behavioral persistence is high in smoking avoidance and cholesterol testing, moderate in influenza vaccination, mammography, and prostate cancer screening, and low for routine exercise and cervical cancer screening. For some of the behaviors we examined, for example, cholesterol testing and cancer screening, there are sound clinical reasons why certain older individuals are not tested routinely. The US Preventive Services Task Force (USPSTF) recommends lipid screening only every 5 years, unless there is prior evidence of elevated lipid levels. Repeated screening is less important in older people because lipid levels are less likely to increase after age 65, although the elderly are at greater absolute risk compared with younger adults [32]. Recommendations with respect to cancer screening depend on expected yield and the impact of early detection on disease treatment and outcomes. For example, the USPTF recommends that women over 40 receive mammograms every 1 - 2 years [25], while both the UPSPTF and the American Cancer Society recommend against routine cervical cancer screening in women over 65 if they have had adequate recent screening with normal Pap smears and are not otherwise at high risk for cervical cancer [26,33]. With respect to prostate cancer screening the USPSTF is uncertain about whether there is in fact a net benefit in men under 75, and in men 75 years and over considers that the harms of screening actually outweigh the benefits [34]. Moreover as the yield of screening declines rapidly with repeated annual testing the USPTF has suggested PSA screening as infrequently as every 4 years may provide as much benefit as annual screening. A possible explanation for the decrease in weekly exercise as age increases is the loss of functionality from comorbidities and frailty.
The various recommendations for screening frequency suggest that measuring persistence over time is a more reliable way to appraise behavior compared to the oneperiod measures typically found in the literature. That said, the factors we found to be most predictive of behavioral persistence among Medicare beneficiaries-higher income, being married and attitudes about seeing a doctor - are similar to those identified in previous research using point-in-time estimates [9-11,15-17]. Our findings with respect to race both complement and differ from previous research. Similar to previous studies, we found that blacks were significantly less likely to be immunized against influenza compared to whites $[9,12,16]$. But we also found that black women had higher rates of mammograms and black men had higher rates of prostate cancer screening than whites, even when controlling for a history of breast or prostate cancer, which contrasts to previous research $[17,18]$.

For several of the health behaviors we analyzed, MCBS interviewers asked respondents to indicate specific reasons why they did not practice the behavior or undergo the test in question. We could not use this information in our main models because of the asymmetric questionnaire design; however, the responses are revealing, and may help inform policies to improve use of preventive services and disease screening. Among MCBS respondents in 2005 (the latest year in our dataset) who reported not getting an influenza vaccination, the top 3 reasons cited were: vaccine unavailable (24\%), didn't know it was needed (19\%), and thought the vaccine could cause influenza (13\%) [36]. Respondents cited two primary reasons for not having cancer screening in 2005: first, that it was not needed or nothing was wrong ( $22 \%$ for mammograms, $21 \%$ for pap smears, and $40 \%$ for PSA testing), and second, that their doctor did not prescribe or recommend it (21\% for mammograms, $27 \%$ for pap smears, and $35 \%$ for PSA tests). Fewer than $3 \%$ of these respondents indicated that their doctors recommended against having the test. In relation to the weekly exercise behavior there were unfortunately no further questions relayed to the respondent to elicit possible reasons for lack of activity.

Such varied responses indicate that there is likely no single or simple answer to optimizing healthy behaviors in the Medicare population. Based on our results, we can definitively conclude that covering a service under Medicare, whether subject to a $20 \%$ Part B coinsurance (e.g., mammography), or without cost sharing (e.g., influenza vaccination and PSA lab test), does not assure high rates of persistence. A systematic literature review of the effectiveness of interventions designed to increase the utilization of Medicare preventive services conducted by RAND [20] concluded that organizational change (such as standing orders for influenza vaccination), patient financial incentives, and patient reminders all showed some 
success in improving utilization of various preventive and screening services among the elderly. Interventions involving multiple tools were found to be more successful than those relying on a single intervention.

Chronic disease accounts for a disproportionately large share of Medicare expenditure and is driven in large part by hospital admissions and readmissions [37,38]. The recent study by Peikes and colleagues [39] evaluating the Medicare Coordinated Care Demonstration project-designed to determine whether enhanced care coordination could improve the quality of care or reduce Medicare expenditures for participating beneficiaries with serious chronic health conditions - is a sobering lesson in just how big a challenge this will be. None of fifteen programs participating in the project reduced regular Medicare expenditures, and in two programs the intervention group actually had higher expenditures, suggesting that care coordination alone is unlikely to be an effective means of reducing total Medicare expenditures for beneficiaries with chronic illnesses.

These results suggest that the need for cost-effective, large-scale, prevention-focused interventions is even more compelling. The sheer size and heterogeneity of the Medicare population makes the task of designing these truly daunting, and finding effective ways of encouraging healthy behaviors among beneficiaries with low socioeconomic status and poor health will be particularly challenging. Nevertheless if Medicare is to be truly sustainable, the need to find ways to reduce the chronic disease burden among Medicare beneficiaries while simultaneously containing costs, is overwhelming.

\section{ACKNOWLEDGEMENTS}

The authors would like to acknowledge J. Samantha Shoemaker, graduate student from the University of Maryland, for her contributions.

\section{REFERENCES}

[1] Christmas, C. and Andersen, R.A. (2000) Exercise and older patients: Guidelines for the clinician. Journal of the American Geriatrics Society, 48, 318-324.

[2] Aldana, S.G. (2001) Financial impact of health promotion programs: A comprehensive review of the literature. American Journal of Health Promotion, 15, 296-320.

[3] Bean, J.F., Vora, A. and Frontera, W.R. (2004) Benefits of exercise for community-dwelling older adults. Archives of Physical Medicine and Rehabilitation, 85, S31-S42. doi:10.1016/j.apmr.2004.03.010

[4] Penedo, F.J., Schneiderman, N., Dahn, J.R. and Gonzalez, J.S. (2004) Physical activity interventions in the elderly: Cancer and comorbidity. Cancer Investigation, 22, 51-67. doi:10.1081/CNV-120027580

[5] Au, D.H., et al. (2009) The effects of smoking cessation on the risk of chronic obstructive pulmonary disease exacerbations. Journal of General Internal Medicine, 24, 457-463. doi:10.1007/s11606-009-0907-y

[6] Hall, K.M. and Luepker, R.V. (2000) Is hypercholesterolemia a risk factor and should it be treated in the elderly? American Journal of Health Promotion, 14, 347-356.

[7] Hak, E., et al. (2002) Influence of high-risk medical conditions on the effectiveness of influenza vaccination among elderly members of 3 large managed-care organizations. Clinical Infectious Disease, 35, 370-377. doi:10.1086/341403

[8] Fiscella, K., Dressler, R., Meldrum, S. and Holt, K. (2007) Impact of influenza vaccination disparities on elderly mortality in the United States. Preventive Medicine, 45, 83-87. doi:10.1016/j.ypmed.2007.03.007

[9] Sambamoorthi, U. and Findley, P.A. (2005) Who are the elderly who never receive influenza immunization? Preventive Medicine, 40, 469-478. doi:10.1016/i.ypmed.2004.07.017

[10] Mark, T.L. and Paramore, L.C. (1996) Pneumococcal pneumonia and influenza vaccination: Access to and use by US Hispanic Medicare beneficiaries. American Journal of Public Health, 86, 1545-1550. doi:10.2105/AJPH.86.11.1545

[11] Bonito, A.J., Lenfestey, N.F., Eicheldinger, C., Iannacchione, V.G. and Campbell, L. (2004) Disparities in immunizations among elderly Medicare beneficiaries, 2000 to 2002. American Journal of Preventive Medicine, 27, 153-160. doi:10.1016/j.amepre.2004.04.004

[12] Xakellis, G.C. (2005) Predictors of influenza immunization in persons over age 65. The Journal of the American Board of Family Medicine, 18, 426-433. doi:10.3122/jabfm.18.5.426

[13] German, P.S., et al. (1995) Extended coverage for preventive services for the elderly: Response and results in a demonstration population. American Journal of Public Health, 85, 379-386. doi:10.2105/AJPH.85.3.379

[14] Williams, S.J., Elder, J.P., Seidman, R.L. and Mayer, J.A. (1997) Preventive services in a Medicare managed care environment. Journal of Community Health, 22, 417-434. doi:10.1023/A:1025176531596

[15] Ozminkowski, R.J., et al. (2006) Predictors of preventive service use among Medicare beneficiaries. Health Care Financing Review, 27, 5-23.

[16] Chen, J.Y., Diamant, A., Pourat, N. and Kagawa-Singer, M. (2005) Racial/ethnic disparities in the use of preventive services among the elderly. American Journal of Preventive Medicine, 29, 388-395. doi:10.1016/j.amepre.2005.08.006

[17] Gilligan, T., Wang, P. and Levin, R. (2004) Racial differences in screening for prostate cancer in the elderly. Archives of Internal Medicine, 164, 1858-1864. doi:10.1001/archinte.164.17.1858

[18] Gornick, M.E., et al. (1996) Effects of race and income on mortality and use of services among Medicare beneficiaries. New England Journal of Medicine, 335, 791-799. doi:10.1056/NEJM199609123351106

[19] Parker, J., Sabogal, F. and Gerbretsadik, T. (1999) Rela- 
tionship between earlier and later mammography screening-California Medicare, 1992 through 1994. The Western Journal of Medicine, 170, 25-27.

[20] Shekelle, P.G., et al. (2003) Interventions that increase the utilization of medicare-funded preventive services for persons age 65 and older. RAND Corporation, Santa Monica.

[21] Picone, G., Brown, D. and Sloan, F. (2004) Do routine eye exams improve vision? International Journal of Health Care Finance and Economics, 4, 43-63. doi:10.1023/B:IHFE.0000019262.27436.3d

[22] Weiss, L.J. and Blustein, J. (1996) Faithful patients: The effect of long-term physician-patient relationship on the costs and use health care by older Americans. American Journal of Public Health, 86, 1742-1747. doi:10.2105/AJPH.86.12.1742

[23] De Bruijn, I.A., et al. (1997) Annually repeated influenza vaccination improves humoral responses to several influenza virus strains in healthy elderly. Vaccine, 15, 1323-1329. doi:10.1016/S0264-410X(97)00019-4

[24] Voordouw, A.C.G., et al. (2004) Annual revaccination against influenza and mortality risk in communitydwelling elderly persons. The Journal of the American Medical Association, 292, 2089-2095. doi:10.1001/jama.292.17.2089

[25] US Preventive Services Task Force (2009) Clinical guidelines for breast cancer screening. http://www.ahrq.gov/clinic/uspstf/uspsbrca.htm

[26] US Preventive Services Task Force (2009) Clinical guidelines for cervical cancer screening. http://www.ahrq.gov/clinic/uspstf/uspscerv.htm

[27] Katz, S. (1983) Assessing self maintenance: Activities of daily living, mobility and instrumental activities of daily living. Journal of the American Geriatrics Society, 31, 721-726.

[28] Stuart, B., Doshi, J. and Terza, J. (2009) Assessing the impact of drug use on hospital costs. Health Services Research, 44, 128-144. doi:10.1111/j.1475-6773.2008.00897.x

[29] Zuckerman, I., et al. (2008) Racial and ethnic disparities in treatment of dementia among Medicare beneficiaries. The Journals of Gerontology, Series B, Psychology Sciences and Social Sciences, 63, S328-S333.
[30] Simoni-Wastila, L., Zuckerman, I., Shaffer, T., Blanchette, C. and Stuart, B. (2008) Drug use patterns in severely mentally ill Medicare beneficiaries: Impact of discontinuities in drug coverage. Health Services Research, 43, 496-514. doi:10.1111/j.1475-6773.2007.00779.x

[31] Gameroff, M.J. (2005) Using the proportional model for health-related outcomes: Why, when, and how with various SAS ${ }^{\circledR}$ procedures. SUGI 30 Proceedings Paper, 205-230.

[32] US Preventive Services Task Force (2009) Clinical guidelines for screening for lipid disorders in adults. http://www.ahrq.gov/clinic/uspstf08/lipid/lipidrs.htm

[33] American Cancer Society (2009) American Cancer Society guidelines for the early detection of cancer. http://www.cancer.org/docroot/PED/content/PED_2_3X ACS_Cancer_Detection_Guidelines_36.asp?sitearea $=\mathrm{PE}$ $\underline{\mathrm{D}}$

[34] US Preventive Services Task Force (2009) Clinical guidelines for prostate cancer screening. http://www.ahrq.gov/clinic/uspstf/uspsprca.htm

[35] US Preventive Services Task Force (2009) Clinical guidelines for colorectal cancer screening. http://www.ahrq.gov/clinic/uspstf/uspscolo.htm

[36] Center for Medicare \& Medicaid Services (2009) Medicare Current Beneficiary Survey Ric 2P. http://www.cms.hhs.gov/MCBS/Downloads/A05\%20Ric \%202P.pdf

[37] Thorpe, K.E. and Howard, D.H. (2006) The rise in spending among Medicare beneficiaries: The role of chronic disease prevalence and changes in treatment intensity. Health Affairs (Millwood), 25, w378-w388. doi:10.1377/hlthaff.25.w378

[38] Medicare Payment Advisory Commission (2008) A databook: Healthcare spending and the medicare program. Medicare Payment Advisory Commission, Washington DC.

[39] Peikes, D., Chen, A., Schore, J. and Brown, R. (2009) Effects of care coordination on hospitalization, quality of care, and health care expenditures among Medicare beneficiaries: 15 randomized trials. The Journal of the American Medical Association, 301, 603-618. doi:10.1001/jama.2009.12 